\title{
Eye Tracking Gets Complex
}

\author{
Two research teams have used eye-tracking methods to learn how \\ students approach complex physics problems.
}

\section{By Katherine Wright}

U sing eye-tracking technology, two research teams studied the process by which students solve complex physics problems and found that both the type of problem and the competence of the student affect the way that the student approaches the problem [1,2]. The technology is not new in physics education research (PER), but it had not previously been used with physics problems that involve understanding multiple concepts or interpreting both diagrams and equations. The results could inform teaching of problem-solving skills.

Eye movements provide a convenient way to track a person's attention. Technologies developed for eye tracking typically involve recording video of a person's eyes and then analyzing the footage to determine where they are looking at each

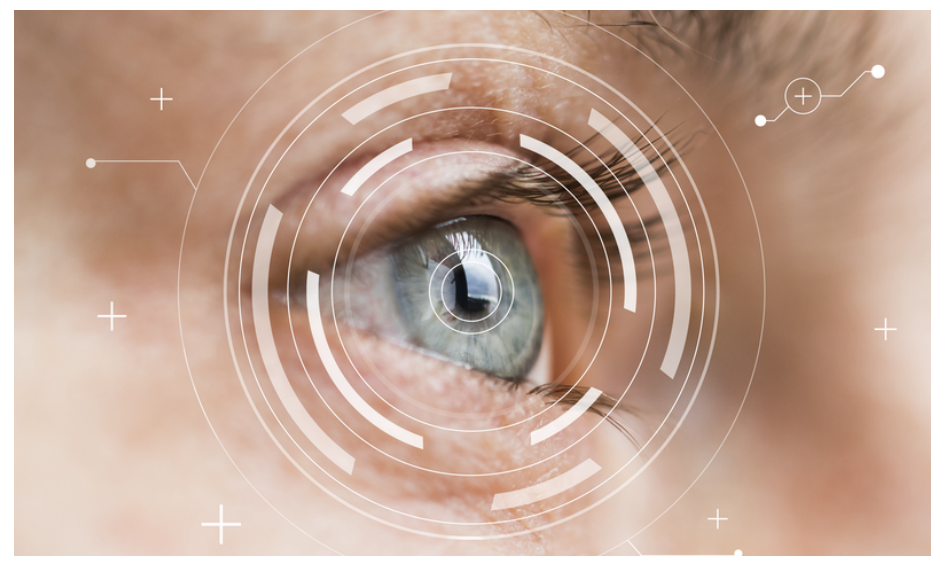

Flickering Eyes. The results from new eye tracking experiments, which monitor the motion of the eyes of physics students as they solve complex problems, could inform the teaching of problem-solving skills. (See example problems below.) Credit: maxsim/stock.adobe.com moment. PER researchers have been using these techniques for about a decade to investigate students' approaches to problem solving. However, most of this work has looked at problems involving a single concept, rather than the multiconcept problems that are an essential part of physics courses. Two research teams have now applied eye tracking to more complicated problems.

In one study, Bashirah Ibrahim of the University of Bahrain and Lin Ding of The Ohio State University tracked the eye motions of 22 students while they worked on four questions. Two of these questions required analyzing a series of chronological events, for example, calculating a roller coaster's energy and speed as it descends and then climbs. The other two questions involved simultaneous events, for example, finding the translational and rotational momentum of a rod at the moment that it is hit by a bullet. All of the problems involved applying knowledge of at least two physics concepts and required that the student study both the wording of the question and a diagram. The students' eyes were tracked twice while they solved each problem: first during a "thinking" phase, when they silently tackled the problem in their heads, and then during a "talking" phase, when they spoke aloud about their methodology.

Ibrahim and Ding found that, when solving the simultaneous problems, the students' eyes moved back and forth between the text and the diagram more frequently than when solving the sequential ones. For sequential problems, students spent more time focused on the diagrams than for simultaneous problems. The team found no link between the eye-movement pattern and whether the student correctly answered the question.

Based on the eye-tracking data and on the students' comments during the talking phase of the study, the researchers hypothesize that students often misinterpreted the 


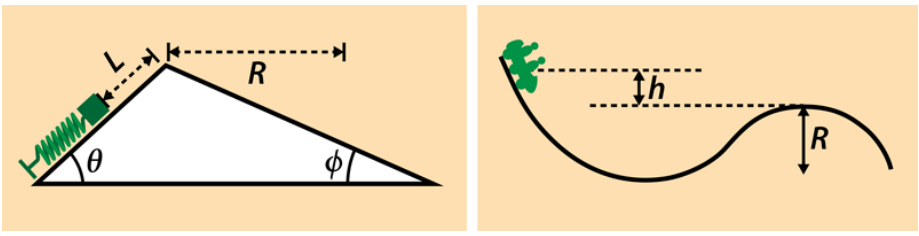

Back to school. These diagrams accompanied the sequential problems used by Ibrahim and Ding. (Left) A spring launches a block over a ridge. Find the distance $R$ from the peak to the place where the block hits the slope, given all parameters, including the mass, the spring stiffness, and the distance the spring is compressed before release. (Right) A roller coaster cart on a frictionless track approaches a semicircular hill. What is the normal force it experiences at the top of the hill, given its initial speed, mass, and initial height $(h)$ above the crest of the hill?

Credit: B. Ibrahim and L. Ding; adapted by APS/Alan Stonebraker

simultaneous problems as single events, not grasping their multistage nature. The observed frequent switching between text and diagram in these problems may have been a result of students needing help interpreting the diagrams, the researchers suggest. For sequential problems, the longer times spent focused on the diagrams may have occurred as students worked out the multiple stages that they correctly perceived.

The bottom line is that different types of problems lead to different student approaches and therefore require different instructional techniques, Ibrahim says. "We cannot indiscriminately lump every problem into the same basket."

In a second study, Chao-Jung Wu and Chia-Yu Liu of the National Taiwan Normal University investigated the effect of students' abilities. They administered a graph-reading test to about 200 students and invited those scoring in either the top third or the bottom third to participate in the eye-tracking study, enrolling 96 of them. The students were given four questions that each required them to interpret information and data presented in four different formats: a written question, a data table, a graph, and an equation. The students were then recorded speaking their answers out loud while their eyes were tracked.

Wu and Liu found a clear link between a student's eye movements and their graph-reading competency. The eyes of those in the high competency group frequently flitted back and forth between the four elements of the question, suggesting an ability to understand and integrate all of the different representations of the information. In contrast, those in the low competency group tended to stick mainly with the text and the table.

The observation by Ibrahim and Ding that students have the same eye-movement patterns-regardless of whether they can solve the problem-is "surprising," given that it conflicts with previous findings for single-concept problems, says PER researcher Tianlong Zu of Lawrence University, Wisconsin. PER researcher Pascal Klein, of the University of Göttingen, Germany, agrees. Klein is less surprised by the study of Wu and Liu, since expertise and visual attention have been linked in previous research on simpler problems. But he notes that the results help pinpoint where students struggle. Clearly, students with lower physics competency need better instruction on how to simultaneously synthesize data from multiple sources. That skill is becoming increasingly important in today's data-driven world, he says.

Katherine Wright is the Deputy Editor for Physics.

\section{REFERENCES}

1. B. Ibrahim and L. Ding, "Sequential and simultaneous synthesis problem solving: A comparison of students' gaze transitions," Phys. Rev. Phys. Educ. Res. 17, 010126 (2021).

2. C. Wu and C. Liu, "Eye-movement study of high- and low-prior-knowledge students' scientific argumentations with multiple representations," Phys. Rev. Phys. Educ. Res. 17, 010125 (2021). 